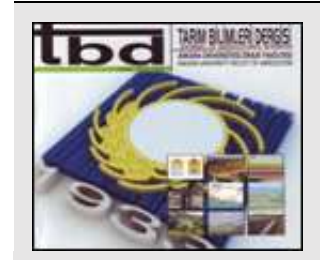

Tarım Bilimleri Dergisi Tar. Bil. Der.

Dergi web sayfas1: www.agri.ankara.edu.tr/dergi
Journal of Agricultural Sciences

Journal homepage: www.agri.ankara.edu.tr/journal

\title{
The Use of an Alternative Differential Set for Determination of Pyrenophora teres f. maculata Pathotypes
}

\author{
Bermet BEISHENKANOVA ${ }^{\text {a }}$ Aziz KARAKAYA ${ }^{\text {a }}$, Arzu ÇELİK OĞUZ

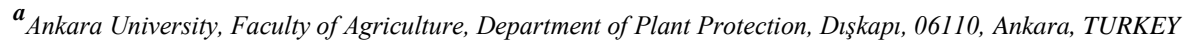

\section{ARTICLE INFO}

Research Article

Corresponding Author: Aziz KARAKAYA, E-mail: karakaya@agri.ankara.edu.tr, Tel: +90 (312) 5961258

Received: 28 December 2018, Received in Revised Form: 24 June 2019, Accepted: 27 June 2019

\section{AUTHORS ORCID ID:}

(Bermet BEISHENKANOVA: 0000-0002-7623-0094), (Aziz KARAKAYA: 0000-0003-3019-9009), (Arzu ÇELIK OĞUZ: 0000-00020906-6407)

\section{ABSTRACT}

Pyrenophora teres f. maculata incites spot form of barley net blotch disease. For determination of Pyrenophora teres f. maculata pathotypes, a differential set consisted of 22 international cultivars and genotypes and a susceptible Turkish barley variety Bülbül 89 were tested using 45 isolates obtained from different regions of Turkey. Nineteen pathotypes were determined out of 45 isolates used. It appears that this differential set could be useful for determination of P. teres f. maculata pathotypes.

\section{Introduction}

Barley net blotch disease caused by the fungus Pyrenophora teres (anamorph: Drechslera teres) is a common and important disease which lowers the yield and quality of the barley in the world (Mathre 1982; McLean et al 2009; Karakaya et al 2014). Pyrenophora teres has two biotypes. P. teres f. maculata and P. teres f. teres incite spot and net forms of net blotch disease, respectively (Liu et al 2011). Resistant cultivars are preferred in disease control. However, pathotypes of the fungus complicate the resistance studies. In order to control the pathogen, information about the pathotypes of the fungus is necessary. For pathotype determination studies, different researchers used different cultivars and genotypes. However, most of the time, comparison of these pathotypes were difficult (Wu et al 2003; Grewal et al 2008; Boungab et al 2012; McLean et al 2014a; McLean et al 2014b). This study aimed at contributing to development of an international set for determination of Pyrenophora teres f. maculata (Ptm) pathotypes.

\section{Material and Methods}

This study was carried out in laboratory and greenhouse of Plant Protection Department of Faculty of Agriculture, Ankara University, Turkey.

Between 2015-2017 surveys were conducted in various provinces of Turkey and 1, 6, 5, 3, 1, 6, 2, 5, 2, 1, 2, 1, 1, 2, 1, 1, 2, 1, 1 and 1 P. teres f. maculata samples were obtained from Niğde, Diyarbakır, Ankara, Eskişehir, Adıyaman, Konya, Kırşehir, Şanlıurfa, Kayseri, Afyonkarahisar, Kahramanmaraş, Kırıkkale, Aksaray, Çankırı, Sivas, Yozgat, Mardin, Kilis, Edirne and Gaziantep provinces of Turkey, respectively. For obtaining samples, barley planting areas in 
each location were considered. In surveys, systematic sampling method was used (Aktaş 2001). Samples were obtained from a diverse set of provinces. Leaves showing characteristic spot form of net blotch symptoms were selected. These leaves were subjected to surface sterilization using $1 \% \mathrm{NaOCl}$ for 1 minute and they were kept in blotter for 4-5 days. Under a stereomicroscope, single spores were taken and transferred to Potato Dextrose Agar plates. From diseased barley and wild barley (Hordeum spontaneum) plants 45 Ptm single spore isolates were obtained. Typical Pyrenophora teres f. maculata conidia were observed in a light microscope. Symptom morphologies of these isolates were verified using the susceptible barley cultivar Bülbül 89 (Mathre 1982; Çelik Oğuz \& Karakaya 2017).

Barley differential cultivars and genotypes Chebec, Haruna Nijo, CI3576, Torrens, Keel, TR250, CI9214, Galleon, CI9819, CI11458, CI5286, CI5791, CI7584, CI9776, CI16150, Skiff, Steptoe, Kombar, Cape, Stirling, Summitt and Arimont were obtained from Mark S. McLean (Agriculture Victoria, Horsham, Australia). In addition, susceptible Turkish cultivar Bülbül 89 (Çelik Oğuz \& Karakaya 2017) was added to the set.

Under greenhouse conditions, differential set genotypes were planted in plastic pots, $7 \mathrm{~cm}$ in diameter, containing topsoil. Each pot contained 5-10 seeds. There were three replications arranged in a completely randomized fashion. Ten days old cultures grown on Potato Dextrose Agar were used as inoculum. Fungal cultures were scraped using a paintbrush and washed through cheesecloth with water. Inoculum which consisted of mycelium pieces, was adjusted to 1.5-2.0x $10^{5}$ mycelium parts per mL. For each $100 \mathrm{~mL}$ of inoculum suspension, one drop of Tween 20 was added (Aktaş 1995). Inoculation of the barley seedlings were performed at the two to three leaf stages (Z12-13; Zadoks et al 1974). Fungal suspensions were sprayed onto barley differential set seedlings. Inoculated plants were kept in closed transparent lid boxes covered by transparent nylon covers for $72 \mathrm{~h}$ in a greenhouse at high humidity. The nylons were then removed and ventilation lids were opened for another $24 \mathrm{~h}$. The temperature of the greenhouse was $18 \pm 1-23 \pm 1{ }^{\circ} \mathrm{C}$ during night and day with a $14 \mathrm{~h} / 10 \mathrm{~h}$ light/dark period. Following this period, box lids were opened. Seven days after inoculation, barley seedlings were assessed for disease severity using the spot form scale described by Tekauz (1985).

For pathotype determination, methods outlined in Wu et al (2003) and Çelik Oğuz \& Karakaya (2017) were used. Seven days later following inoculation, plants were evaluated using a 1-9 scale developed by Tekauz (1985). For evaluation, second leaves were used. Scale values between 1-5 and 6-9 were considered as resistant and susceptible, respectively. Differential test genotypes were numbered 1 through 23 and pathotypes were determined according to their responses to these differential set genotypes. For example, isolate PTM 42 from Yozgat province showed susceptible reactions (>5) on genotypes 13 (Galleon), 18 (Steptoe) and 19 (Stirling) and showed resistant reactions $(\leq 5)$ on the other differential set genotypes. Therefore, this pathotype was named as 13-18-19. Isolates exhibiting resistant reactions $(\leq 5)$ to all differential test genotypes were termed as pathotype 0 .

\section{Results and Discussion}

The scale values of 45 isolates ranged between 1-8 (Figure 1). Nineteen pathotypes were determined using 45 Ptm isolates based on their differential reactions to 23 barley genotypes (Tables 1 and 2). No genotype was either resistant or susceptible to all isolates. Genotypes Chebec, CI5286, CI7584, CI9819 and CI16150 exhibited resistant reactions to 43 isolates (95.5\%). These genotypes were susceptible to only 2 isolates. Genotypes Arimont, CI5791, Skiff and TR250 showed resistant reactions to 42 isolates (93\%). Genotypes CI3576, CI9214, CI9776 and Torrens exhibited resistant reactions to $91 \%$ of the isolates (41 isolates). Genotypes Cape, Keel, Galleon, Haruna Nijo, Kombar, Summitt, CI11458 and Stirling showed resistant reactions to $88 \%, 86 \%, 84 \%, 84 \%, 82 \%, 82 \%, 80$ and $80 \%$ of the isolates, respectively. Cultivar Steptoe exhibited susceptible reaction to 18 isolates (40\%) and cultivar Bülbül 89 showed susceptible reaction to 19 isolates $(42 \%)$.

In previous studies, different differential test genotypes were used by different researchers. McLean et al (2014b) performed a study between 2008-2013 in Australia, South Africa, Finland and Canada and developed a new Ptm differential set. This set consisted of Arimont, Baudin, Beecher, Cape, Chebec, CI11458, CI3576, CI5286, CI5791, CI7584, CI9214, CI9776, CI9819, CI9831, CI16150, Galleon, Haruna Nijo, Keel, Kombar, Skiff, Steptoe, Stirling, Summitt, Torrens, TR250 and Yagan cultivars and genotypes. In this study, virulence diversity among the isolates was observed. Among these barley genotypes Arimont, Cape, Chebec, CI11458, CI5286, CI5791, CI7584, CI9214, CI9776, CI9819, CI16150, Galleon, Haruna Nijo, Keel, Kombar, Skiff, Steptoe, Stirling, Summitt, Torrens and TR250 were also used in our current study and it is concluded that these genotypes could be used as Ptm differential test genotypes. 

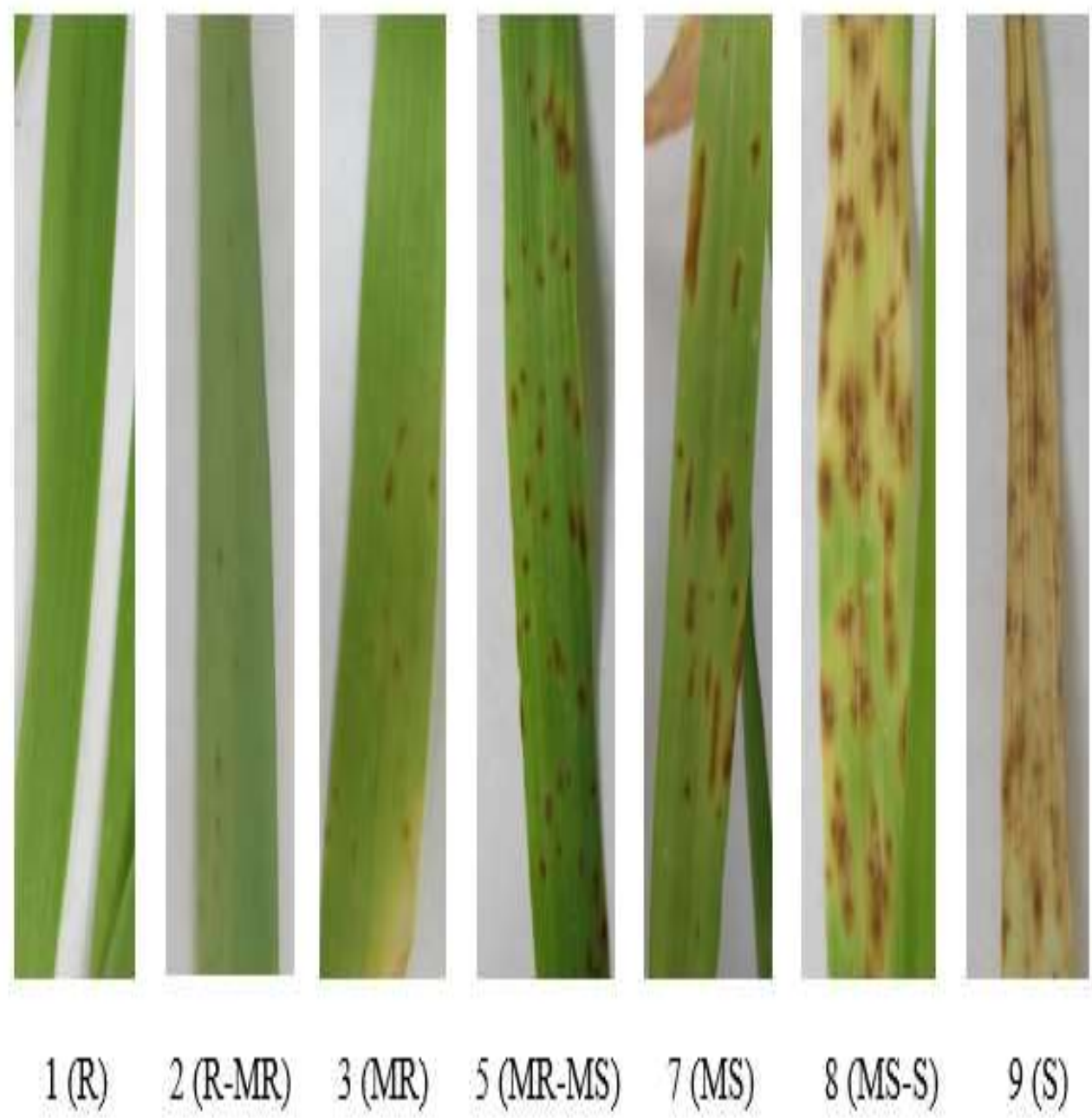

Figure 1- Reactions of barley differential set genotypes to Pyrenophora teres f. maculata isolates according to Tekauz (1985) scale; R, resistant; R-MR, resistant-moderately resistant; MR, moderately resistant; MR-MS, moderately resistant-moderately susceptible; MS, moderately susceptible; MS-S, moderately susceptible-susceptible; S, susceptible

Using 11 differential set genotypes, Akhavan et al (2016) identified 13 pathotype groups out of 27 isolates used. Two groups contained $52 \%$ of the isolates. Wu et al (2003) used a differential set containing 25 barley genotypes. In their study, 4 pathotypes were distinguished among the 8 isolates. Tekauz (1990) used 11 barley differential set genotypes. From 42 isolates 20 pathotypes were distinguished. Using 16 differential set genotypes and 60 Ptm isolates, McLean et al (2014a) determined 33 pathotypes.

In Turkey, Çelik Oğuz \& Karakaya (2017) used 25 differential set genotypes. From a total of 50 isolates, 26 Ptm pathotypes were determined. In our current study, we used 23 differential set genotypes and from a total of 45 isolates, 19 pathotypes were distinguished. Karki \& Sharp (1986) used a differential set which consisted of 20 genotypes. In their study, 6 groups were evident among the 14 isolates used. Gupta et al (2012) used a differential set which consisted of 26 genotypes. In their study, 7 groups were found among the 49 isolates used.

In our current study, differential genotypes Chebec, CI5286, CI7584, CI9819 and CI16150 exhibited resistant reactions to 43 isolates $(95.5 \%)$ and susceptible reactions to 2 isolates. Karki \& Sharp (1986), using isolates obtained from Montana (USA) and other countries, reported different reactions on genotypes CI7584 and CI9819. McLean et al (2012) reported Chebec and CI16150 genotypes as moderately resistant. McLean et al (2014a) reported different reactions of the genotype CI5286 to the isolates. 
Table 1- Response of barley differential genotypes to 45 Pyrenophora teres f. maculata isolates. For evaluation, a 1-9 scale developed by Tekauz (1985) was used. Numbers are mean of 3 replications. $R$, resistant; $S$, susceptible

\begin{tabular}{|c|c|c|c|c|c|c|c|c|c|c|c|c|c|c|c|c|c|c|}
\hline $\begin{array}{l}\text { Barley } \\
\text { genotypes }\end{array}$ & $\underset{\mathbb{Z}}{\mathbb{Z}}$ & 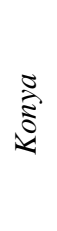 & $\underset{k}{N}$ & 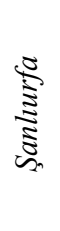 & & & $\underset{⿱ 乛}{\mathbb{Z}}$ & 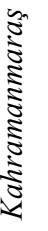 & 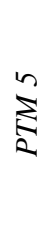 & & $\sum_{k}^{b}$ & $\frac{\Sigma}{3}$ & $\sum_{k}^{N}$ & 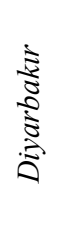 & $\underset{\Sigma}{\infty}$ & 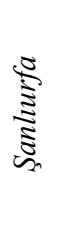 & 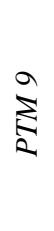 & 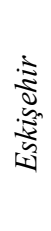 \\
\hline 1 Arimont & 5 & $\mathrm{R}$ & 3 & $\mathrm{R}$ & 3 & $\mathrm{R}$ & 5 & $\mathrm{R}$ & 5 & $\mathrm{R}$ & 5 & $\mathrm{R}$ & 1 & $\mathrm{R}$ & 2 & $\mathrm{R}$ & 1 & $\mathrm{R}$ \\
\hline 2 Cape & 3 & $\mathrm{R}$ & 3 & $\mathrm{R}$ & 3 & $\mathrm{R}$ & 5 & $\mathrm{R}$ & 3 & $\mathrm{R}$ & 5 & $\mathrm{R}$ & 3 & $\mathrm{R}$ & 1 & $\mathrm{R}$ & 3 & $\mathrm{R}$ \\
\hline 3 Chebec & 1 & $\mathrm{R}$ & 2 & $\mathrm{R}$ & 1 & $\mathrm{R}$ & 3 & $\mathrm{R}$ & 2 & $\mathrm{R}$ & 3 & $\mathrm{R}$ & 1 & $\mathrm{R}$ & 2 & $\mathrm{R}$ & 2 & $\mathrm{R}$ \\
\hline 4 CI3546 & 3 & $\mathrm{R}$ & 3 & $\mathrm{R}$ & 5 & $\mathrm{R}$ & 5 & $\mathrm{R}$ & 5 & $\mathrm{R}$ & 5 & $\mathrm{R}$ & 2 & $\mathrm{R}$ & 2 & $\mathrm{R}$ & 2 & $\mathrm{R}$ \\
\hline 5 CI11458 & 2 & $\mathrm{R}$ & 3 & $\mathrm{R}$ & 3 & $\mathrm{R}$ & 7 & $\mathrm{~S}$ & 3 & $\mathrm{R}$ & 7 & $\mathrm{~S}$ & 2 & $\mathrm{R}$ & 2 & $\mathrm{R}$ & 1 & $\mathrm{R}$ \\
\hline 6 CI5286 & 3 & $\mathrm{R}$ & 2 & $\mathrm{R}$ & 1 & $\mathrm{R}$ & 5 & $\mathrm{R}$ & 2 & $\mathrm{R}$ & 3 & $\mathrm{R}$ & 2 & $\mathrm{R}$ & 1 & $\mathrm{R}$ & 1 & $\mathrm{R}$ \\
\hline 7 CI5791 & 1 & $\mathrm{R}$ & 1 & $\mathrm{R}$ & 5 & $\mathrm{R}$ & 5 & $\mathrm{R}$ & 2 & $\mathrm{R}$ & 5 & $\mathrm{R}$ & 1 & $\mathrm{R}$ & 1 & $\mathrm{R}$ & 1 & $\mathrm{R}$ \\
\hline 8 CI7584 & 2 & $\mathrm{R}$ & 3 & $\mathrm{R}$ & 1 & $\mathrm{R}$ & 5 & $\mathrm{R}$ & 2 & $\mathrm{R}$ & 5 & $\mathrm{R}$ & 1 & $\mathrm{R}$ & 2 & $\mathrm{R}$ & 2 & $\mathrm{R}$ \\
\hline 9 CI9214 & 2 & $\mathrm{R}$ & 1 & $\mathrm{R}$ & 5 & $\mathrm{R}$ & 5 & $\mathrm{R}$ & 3 & $\mathrm{R}$ & 3 & $\mathrm{R}$ & 2 & $\mathrm{R}$ & 2 & $\mathrm{R}$ & 2 & $\mathrm{R}$ \\
\hline 10 CI9776 & 1 & $\mathrm{R}$ & 1 & $\mathrm{R}$ & 2 & $\mathrm{R}$ & 5 & $\mathrm{R}$ & 3 & $\mathrm{R}$ & 5 & $\mathrm{R}$ & 1 & $\mathrm{R}$ & 1 & $\mathrm{R}$ & 2 & $\mathrm{R}$ \\
\hline 11 CI9819 & 2 & $\mathrm{R}$ & 3 & $\mathrm{R}$ & 3 & $\mathrm{R}$ & 5 & $\mathrm{R}$ & 2 & $\mathrm{R}$ & 5 & $\mathrm{R}$ & 2 & $\mathrm{R}$ & 2 & $\mathrm{R}$ & 1 & $\mathrm{R}$ \\
\hline 12 CI16150 & 2 & $\mathrm{R}$ & 1 & $\mathrm{R}$ & 3 & $\mathrm{R}$ & 5 & $\mathrm{R}$ & 5 & $\mathrm{R}$ & 3 & $\mathrm{R}$ & 1 & $\mathrm{R}$ & 2 & $\mathrm{R}$ & 2 & $\mathrm{R}$ \\
\hline 13 Galleon & 3 & $\mathrm{R}$ & 1 & $\mathrm{R}$ & 2 & $\mathrm{R}$ & 5 & $\mathrm{R}$ & 3 & $\mathrm{R}$ & 2 & $\mathrm{R}$ & 1 & $\mathrm{R}$ & 2 & $\mathrm{R}$ & 2 & $\mathrm{R}$ \\
\hline 14 Haruna Nijo & 3 & $\mathrm{R}$ & 2 & $\mathrm{R}$ & 3 & $\mathrm{R}$ & 5 & $\mathrm{R}$ & 5 & $\mathrm{R}$ & 7 & $S$ & 3 & $\mathrm{R}$ & 2 & $\mathrm{R}$ & 2 & $\mathrm{R}$ \\
\hline 15 Keel & 3 & $\mathrm{R}$ & 1 & $\mathrm{R}$ & 3 & $\mathrm{R}$ & 3 & $\mathrm{R}$ & 5 & $\mathrm{R}$ & 3 & $\mathrm{R}$ & 2 & $\mathrm{R}$ & 1 & $\mathrm{R}$ & 1 & $\mathrm{R}$ \\
\hline 16 Kombar & 5 & $\mathrm{R}$ & 1 & $\mathrm{R}$ & 2 & $\mathrm{R}$ & 5 & $\mathrm{R}$ & 3 & $\mathrm{R}$ & 5 & $\mathrm{R}$ & 1 & $\mathrm{R}$ & 2 & $\mathrm{R}$ & 2 & $\mathrm{R}$ \\
\hline 17 Skiff & 3 & $\mathrm{R}$ & 2 & $\mathrm{R}$ & 2 & $\mathrm{R}$ & 3 & $\mathrm{R}$ & 3 & $\mathrm{R}$ & 3 & $\mathrm{R}$ & 1 & $\mathrm{R}$ & 2 & $\mathrm{R}$ & 1 & $\mathrm{R}$ \\
\hline 18 Steptoe & 5 & $\mathrm{R}$ & 3 & $\mathrm{R}$ & 3 & $\mathrm{R}$ & 5 & $\mathrm{R}$ & 5 & $\mathrm{R}$ & 7 & $\mathrm{~S}$ & 2 & $\mathrm{R}$ & 3 & $\mathrm{R}$ & 1 & $\mathrm{R}$ \\
\hline 19 Stirling & 5 & $\mathrm{R}$ & 2 & $\mathrm{R}$ & 5 & $\mathrm{R}$ & 5 & $\mathrm{R}$ & 3 & $\mathrm{R}$ & 7 & $\mathrm{~S}$ & 1 & $\mathrm{R}$ & 2 & $\mathrm{R}$ & 1 & $\mathrm{R}$ \\
\hline 20 Summitt & 3 & $\mathrm{R}$ & 2 & $\mathrm{R}$ & 3 & $\mathrm{R}$ & 7 & $S$ & 2 & $\mathrm{R}$ & 2 & $\mathrm{R}$ & 2 & $\mathrm{R}$ & 1 & $\mathrm{R}$ & 1 & $\mathrm{R}$ \\
\hline 21 Torrens & 3 & $\mathrm{R}$ & 1 & $\mathrm{R}$ & 5 & $\mathrm{R}$ & 5 & $\mathrm{R}$ & 2 & $\mathrm{R}$ & 5 & $\mathrm{R}$ & 2 & $\mathrm{R}$ & 1 & $\mathrm{R}$ & 1 & $\mathrm{R}$ \\
\hline 22 TR250 & 3 & $\mathrm{R}$ & 1 & $\mathrm{R}$ & 3 & $\mathrm{R}$ & 5 & $\mathrm{R}$ & 2 & $\mathrm{R}$ & 3 & $\mathrm{R}$ & 3 & $\mathrm{R}$ & 1 & $\mathrm{R}$ & 1 & $\mathrm{R}$ \\
\hline 23 Bülbül 89 & 7 & $S$ & 3 & $\mathrm{R}$ & 7 & $\mathrm{~S}$ & 7 & $S$ & 7 & $S$ & 5 & $\mathrm{R}$ & 3 & $\mathrm{R}$ & 3 & $\mathrm{R}$ & 3 & $\mathrm{R}$ \\
\hline
\end{tabular}


Table 1 (Continued)- Response of barley differential genotypes to 45 Pyrenophora teres f. maculata isolates. For evaluation, a 1-9 scale developed by Tekauz (1985) was used. Numbers are mean of 3 replications. R, resistant; S, susceptible

Isolate numbers and the provinces where the isolates obtained

\begin{tabular}{|c|c|c|c|c|c|c|c|c|c|c|c|c|c|c|c|c|c|c|}
\hline $\begin{array}{l}\text { Barley } \\
\text { genotypes }\end{array}$ & & 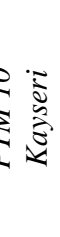 & & $\frac{2}{3}$ & & 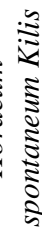 & & 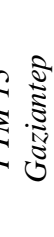 & & 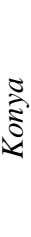 & & & & & 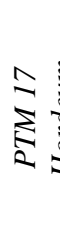 & 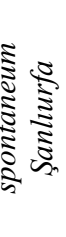 & & ב⿱艹 \\
\hline 1 Arimont & 3 & $\mathrm{R}$ & 5 & $\mathrm{R}$ & 5 & $\mathrm{R}$ & 7 & $\mathrm{~S}$ & 1 & $\mathrm{~F}$ & 3 & $\mathrm{R}$ & 5 & $\mathrm{R}$ & 8 & $\mathrm{~S}$ & 5 & $\mathrm{R}$ \\
\hline 2 Cape & 1 & $\mathrm{R}$ & 7 & S & 5 & $\mathrm{R}$ & 5 & $\mathrm{R}$ & 1 & $\mathrm{r}$ & 2 & $\mathrm{R}$ & 3 & $\mathrm{R}$ & 8 & $\mathrm{~S}$ & 3 & $\mathrm{R}$ \\
\hline 3 Chebec & 2 & $\mathrm{R}$ & 1 & $\mathrm{R}$ & 1 & $\mathrm{R}$ & 7 & S & 1 & $F$ & 3 & $\mathrm{R}$ & 5 & $\mathrm{R}$ & 7 & S & 3 & $\mathrm{R}$ \\
\hline 4 CI3546 & 2 & $\mathrm{R}$ & 7 & $\mathrm{~S}$ & 5 & $\mathrm{R}$ & 5 & $\mathrm{R}$ & 3 & $\mathrm{~F}$ & 3 & $\mathrm{R}$ & 3 & $\mathrm{R}$ & 7 & $S$ & 3 & $\mathrm{R}$ \\
\hline 5 CI11458 & 2 & $\mathrm{R}$ & 7 & $\mathrm{~S}$ & 7 & S & 7 & S & 2 & $F$ & 2 & $\mathrm{R}$ & 5 & $\mathrm{R}$ & 8 & $S$ & 3 & $\mathrm{R}$ \\
\hline 6 CI5286 & 1 & $\mathrm{R}$ & 5 & $\mathrm{R}$ & 5 & $\mathrm{R}$ & 5 & $\mathrm{R}$ & 1 & $\mathrm{~F}$ & 2 & $\mathrm{R}$ & 3 & $\mathrm{R}$ & 7 & $S$ & 3 & $\mathrm{R}$ \\
\hline 7 CI5791 & 1 & $\mathrm{R}$ & 5 & $\mathrm{R}$ & 5 & $\mathrm{R}$ & 7 & S & 3 & $F$ & 3 & $\mathrm{R}$ & 3 & $\mathrm{R}$ & 8 & S & 3 & $\mathrm{R}$ \\
\hline 8 CI7584 & 2 & $\mathrm{R}$ & 3 & $\mathrm{R}$ & 5 & $\mathrm{R}$ & 5 & $\mathrm{R}$ & 1 & $\mathrm{~F}$ & 3 & $\mathrm{R}$ & 2 & $\mathrm{R}$ & 7 & $\mathrm{~S}$ & 2 & $\mathrm{R}$ \\
\hline 9 CI9214 & 1 & $\mathrm{R}$ & 5 & $\mathrm{R}$ & 3 & $\mathrm{R}$ & 5 & $\mathrm{R}$ & 1 & $\mathrm{~F}$ & 2 & $\mathrm{R}$ & 2 & $\mathrm{R}$ & 7 & S & 5 & $\mathrm{R}$ \\
\hline 10 CI9776 & 1 & $\mathrm{R}$ & 5 & $\mathrm{R}$ & 5 & $\mathrm{R}$ & 7 & S & 1 & $\mathrm{~F}$ & 3 & $\mathrm{R}$ & 3 & $\mathrm{R}$ & 7 & S & 7 & $\mathrm{~S}$ \\
\hline 11 CI9819 & 2 & $\mathrm{R}$ & 5 & $\mathrm{R}$ & 5 & $\mathrm{R}$ & 7 & $S$ & 2 & $\mathrm{~F}$ & 2 & $\mathrm{R}$ & 3 & $\mathrm{R}$ & 7 & $S$ & 3 & $\mathrm{R}$ \\
\hline 12 CI16150 & 2 & $\mathrm{R}$ & 5 & $\mathrm{R}$ & 5 & $\mathrm{R}$ & 8 & S & 2 & $\mathrm{~F}$ & 2 & $\mathrm{R}$ & 2 & $\mathrm{R}$ & 7 & S & 5 & $\mathrm{R}$ \\
\hline 13 Galleon & 1 & $\mathrm{R}$ & 3 & $\mathrm{R}$ & 5 & $\mathrm{R}$ & 8 & $S$ & 2 & $\mathrm{~F}$ & 3 & $\mathrm{R}$ & 5 & $\mathrm{R}$ & 7 & $S$ & 7 & $\mathrm{~S}$ \\
\hline 14 Haruna Nijo & 1 & $\mathrm{R}$ & 5 & $\mathrm{R}$ & 7 & $\mathrm{~S}$ & 7 & $S$ & 1 & $\mathrm{~F}$ & 1 & $\mathrm{R}$ & 3 & $\mathrm{R}$ & 8 & $S$ & 5 & $\mathrm{R}$ \\
\hline 15 Keel & 2 & $\mathrm{R}$ & 3 & $\mathrm{R}$ & 5 & $\mathrm{R}$ & 8 & $S$ & 1 & $\mathrm{~F}$ & 3 & $\mathrm{R}$ & 5 & $\mathrm{R}$ & 7 & $S$ & 5 & $\mathrm{R}$ \\
\hline 16 Kombar & 1 & $\mathrm{R}$ & 8 & $\mathrm{~S}$ & 7 & $\mathrm{~S}$ & 8 & $S$ & 3 & $\mathrm{~F}$ & 3 & $\mathrm{R}$ & 3 & $\mathrm{R}$ & 8 & $S$ & 3 & $\mathrm{R}$ \\
\hline 17 Skiff & 2 & $\mathrm{R}$ & 3 & $\mathrm{R}$ & 3 & $\mathrm{R}$ & 7 & $S$ & 2 & $\mathrm{~F}$ & 5 & $\mathrm{R}$ & 5 & $\mathrm{R}$ & 7 & $\mathrm{~S}$ & 5 & $\mathrm{R}$ \\
\hline 18 Steptoe & 1 & $\mathrm{R}$ & 5 & $\mathrm{R}$ & 5 & $\mathrm{R}$ & 8 & $S$ & 2 & $\mathrm{~F}$ & 7 & S & 7 & $S$ & 8 & $S$ & 7 & $\mathrm{~S}$ \\
\hline 19 Stirling & 2 & $\mathrm{R}$ & 7 & $\mathrm{~S}$ & 7 & $S$ & 7 & $S$ & 2 & $\mathrm{~F}$ & 3 & $\mathrm{R}$ & 5 & $\mathrm{R}$ & 8 & $S$ & 5 & $\mathrm{R}$ \\
\hline 20 Summitt & 2 & $\mathrm{R}$ & 7 & $\mathrm{~S}$ & 7 & $\mathrm{~S}$ & 7 & $\mathrm{~S}$ & 2 & $\mathrm{~F}$ & 3 & $\mathrm{R}$ & 3 & $\mathrm{R}$ & 8 & $\mathrm{~S}$ & 5 & $\mathrm{R}$ \\
\hline 21 Torrens & 1 & $\mathrm{R}$ & 5 & $\mathrm{R}$ & 5 & $\mathrm{R}$ & 5 & $\mathrm{R}$ & 2 & $\mathrm{~F}$ & 5 & $\mathrm{R}$ & 5 & $\mathrm{R}$ & 8 & $\mathrm{~S}$ & 5 & $\mathrm{R}$ \\
\hline $22 \mathrm{TR} 250$ & 2 & $\mathrm{R}$ & 5 & $\mathrm{R}$ & 5 & $\mathrm{R}$ & 5 & $\mathrm{R}$ & 1 & $\mathrm{~F}$ & 2 & $\mathrm{R}$ & 2 & $\mathrm{R}$ & 7 & $S$ & 3 & $\mathrm{R}$ \\
\hline 23 Bülbül 89 & 7 & $S$ & 7 & $\mathrm{~S}$ & 5 & $\mathrm{R}$ & 8 & $S$ & 2 & $\mathrm{~F}$ & 7 & $S$ & 8 & $S$ & 8 & $S$ & 8 & $\mathrm{~S}$ \\
\hline
\end{tabular}


Table 1 (Continued)- Response of barley differential genotypes to 45 Pyrenophora teres f. maculata isolates. For evaluation, a 1-9 scale developed by Tekauz (1985) was used. Numbers are mean of 3 replications. R, resistant; S, susceptible

Isolate numbers and the provinces where the isolates obtained

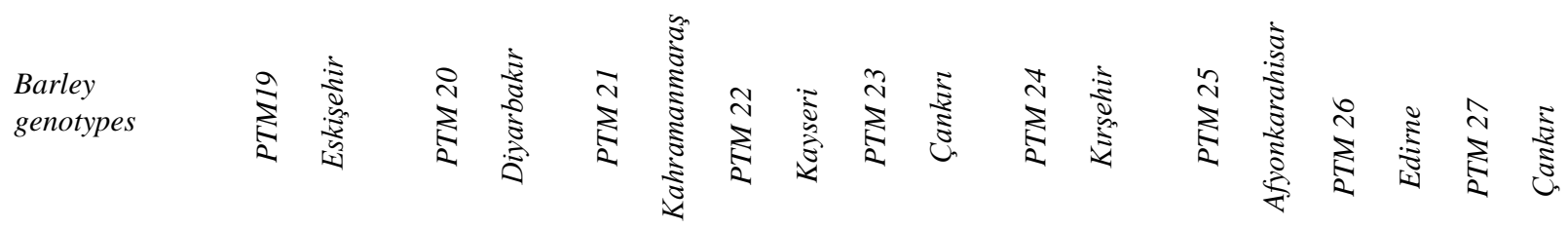

\begin{tabular}{|c|c|c|c|c|c|c|c|c|c|c|c|c|c|c|c|c|c|c|}
\hline 1 Arimont & 2 & $\mathrm{R}$ & 3 & $\mathrm{R}$ & 5 & $\mathrm{R}$ & 1 & $\mathrm{R}$ & 5 & $\mathrm{R}$ & 2 & $\mathrm{R}$ & 2 & $\mathrm{R}$ & 5 & $\mathrm{R}$ & 5 & $\mathrm{R}$ \\
\hline 2 Cape & 2 & $\mathrm{R}$ & 3 & $\mathrm{R}$ & 5 & $\mathrm{R}$ & 1 & $\mathrm{R}$ & 3 & $\mathrm{R}$ & 1 & $\mathrm{R}$ & 2 & $\mathrm{R}$ & 7 & $\mathrm{~S}$ & 3 & $\mathrm{R}$ \\
\hline 3 Chebec & 2 & $\mathrm{R}$ & 2 & $\mathrm{R}$ & 3 & $\mathrm{R}$ & 1 & $\mathrm{R}$ & 3 & $\mathrm{R}$ & 2 & $\mathrm{R}$ & 2 & $\mathrm{R}$ & 5 & $\mathrm{R}$ & 3 & $\mathrm{R}$ \\
\hline $4 \mathrm{CI} 3546$ & 2 & $\mathrm{R}$ & 5 & $\mathrm{R}$ & 5 & $\mathrm{R}$ & 2 & $\mathrm{R}$ & 5 & $\mathrm{R}$ & 2 & $\mathrm{R}$ & 2 & $\mathrm{R}$ & 7 & $\mathrm{~S}$ & 3 & $\mathrm{R}$ \\
\hline 5 CI11458 & 2 & $\mathrm{R}$ & 5 & $\mathrm{R}$ & 5 & $\mathrm{R}$ & 3 & $\mathrm{R}$ & 3 & $\mathrm{R}$ & 2 & $\mathrm{R}$ & 2 & $\mathrm{R}$ & 8 & $\mathrm{~S}$ & 5 & $\mathrm{R}$ \\
\hline 6 CI5286 & 2 & $\mathrm{R}$ & 3 & $\mathrm{R}$ & 3 & $\mathrm{R}$ & 1 & $\mathrm{R}$ & 3 & $\mathrm{R}$ & 1 & $\mathrm{R}$ & 2 & $\mathrm{R}$ & 5 & $\mathrm{R}$ & 3 & $\mathrm{R}$ \\
\hline 7 CI5791 & 2 & $\mathrm{R}$ & 5 & $\mathrm{R}$ & 5 & $\mathrm{R}$ & 3 & $\mathrm{R}$ & 3 & $\mathrm{R}$ & 1 & $\mathrm{R}$ & 2 & $\mathrm{R}$ & 5 & $\mathrm{R}$ & 3 & $\mathrm{R}$ \\
\hline 8 CI7584 & 1 & $\mathrm{R}$ & 5 & $\mathrm{R}$ & 3 & $\mathrm{R}$ & 2 & $\mathrm{R}$ & 3 & $\mathrm{R}$ & 1 & $\mathrm{R}$ & 2 & $\mathrm{R}$ & 5 & $\mathrm{R}$ & 3 & $\mathrm{R}$ \\
\hline 9 CI9214 & 2 & $\mathrm{R}$ & 3 & $\mathrm{R}$ & 2 & $\mathrm{R}$ & 2 & $\mathrm{R}$ & 2 & $\mathrm{R}$ & 2 & $\mathrm{R}$ & 2 & $\mathrm{R}$ & 5 & $\mathrm{R}$ & 5 & $\mathrm{R}$ \\
\hline 10 CI9776 & 1 & $\mathrm{R}$ & 5 & $\mathrm{R}$ & 2 & $\mathrm{R}$ & 1 & $\mathrm{R}$ & 3 & $\mathrm{R}$ & 1 & $\mathrm{R}$ & 1 & $\mathrm{R}$ & 7 & $\mathrm{~S}$ & 3 & $\mathrm{R}$ \\
\hline 11 CI9819 & 2 & $\mathrm{R}$ & 5 & $\mathrm{R}$ & 5 & $\mathrm{R}$ & 2 & $\mathrm{R}$ & 3 & $\mathrm{R}$ & 2 & $\mathrm{R}$ & 1 & $\mathrm{R}$ & 5 & $\mathrm{R}$ & 3 & $\mathrm{R}$ \\
\hline 12 CI16150 & 2 & $\mathrm{R}$ & 5 & $\mathrm{R}$ & 2 & $\mathrm{R}$ & 1 & $\mathrm{R}$ & 3 & $\mathrm{R}$ & 1 & $\mathrm{R}$ & 2 & $\mathrm{R}$ & 5 & $\mathrm{R}$ & 3 & $\mathrm{R}$ \\
\hline 13 Galleon & 1 & $\mathrm{R}$ & 5 & $\mathrm{R}$ & 2 & $\mathrm{R}$ & 2 & $\mathrm{R}$ & 5 & $\mathrm{R}$ & 2 & $\mathrm{R}$ & 1 & $\mathrm{R}$ & 7 & S & 5 & $\mathrm{R}$ \\
\hline 14 Haruna Nijo & 1 & $\mathrm{R}$ & 3 & $\mathrm{R}$ & 5 & $\mathrm{R}$ & 2 & $\mathrm{R}$ & 3 & $\mathrm{R}$ & 1 & $\mathrm{R}$ & 2 & $\mathrm{R}$ & 7 & S & 3 & $\mathrm{R}$ \\
\hline $15 \mathrm{Keel}$ & 3 & $\mathrm{R}$ & 2 & $\mathrm{R}$ & 1 & $\mathrm{R}$ & 1 & $\mathrm{R}$ & 3 & $\mathrm{R}$ & 2 & $\mathrm{R}$ & 1 & $\mathrm{R}$ & 5 & $\mathrm{R}$ & 7 & $\mathrm{~S}$ \\
\hline 16 Kombar & 3 & $\mathrm{R}$ & 5 & $\mathrm{R}$ & 5 & $\mathrm{R}$ & 2 & $\mathrm{R}$ & 3 & $\mathrm{R}$ & 3 & $\mathrm{R}$ & 2 & $\mathrm{R}$ & 7 & S & 7 & $\mathrm{~S}$ \\
\hline 17 Skiff & 3 & $\mathrm{R}$ & 3 & $\mathrm{R}$ & 3 & $\mathrm{R}$ & 3 & $\mathrm{R}$ & 5 & $\mathrm{R}$ & 3 & $\mathrm{R}$ & 1 & $\mathrm{R}$ & 5 & $\mathrm{R}$ & 5 & $\mathrm{R}$ \\
\hline 18 Steptoe & 5 & $\mathrm{R}$ & 5 & $\mathrm{R}$ & 3 & $\mathrm{R}$ & 2 & $\mathrm{R}$ & 7 & S & 3 & $\mathrm{R}$ & 3 & $\mathrm{R}$ & 7 & $\mathrm{~S}$ & 8 & $\mathrm{~S}$ \\
\hline 19 Stirling & 3 & $\mathrm{R}$ & 5 & $\mathrm{R}$ & 5 & $\mathrm{R}$ & 1 & $\mathrm{R}$ & 5 & $\mathrm{R}$ & 2 & $\mathrm{R}$ & 2 & $\mathrm{R}$ & 7 & S & 5 & $\mathrm{R}$ \\
\hline 20 Summitt & 2 & $\mathrm{R}$ & 3 & $\mathrm{R}$ & 3 & $\mathrm{R}$ & 2 & $\mathrm{R}$ & 3 & $\mathrm{R}$ & 3 & $\mathrm{R}$ & 1 & $\mathrm{R}$ & 7 & $\mathrm{~S}$ & 3 & $\mathrm{R}$ \\
\hline 21 Torrens & 1 & $\mathrm{R}$ & 3 & $\mathrm{R}$ & 5 & $\mathrm{R}$ & 2 & $\mathrm{R}$ & 5 & $\mathrm{R}$ & 3 & $\mathrm{R}$ & 2 & $\mathrm{R}$ & 7 & S & 5 & $\mathrm{R}$ \\
\hline 22 TR250 & 1 & $\mathrm{R}$ & 5 & $\mathrm{R}$ & 3 & $\mathrm{R}$ & 2 & $\mathrm{R}$ & 2 & $\mathrm{R}$ & 2 & $\mathrm{R}$ & 1 & $\mathrm{R}$ & 5 & $\mathrm{R}$ & 3 & $\mathrm{R}$ \\
\hline 23 Bülbül 89 & 5 & $\mathrm{R}$ & 5 & $\mathrm{R}$ & 3 & $\mathrm{R}$ & 2 & $\mathrm{R}$ & 7 & S & 7 & $\mathrm{~S}$ & 5 & $\mathrm{R}$ & 5 & $\mathrm{R}$ & 8 & $\mathrm{~S}$ \\
\hline
\end{tabular}


Table 1 (Continued)- Response of barley differential genotypes to 45 Pyrenophora teres f. maculata isolates. For evaluation, a 1-9 scale developed by Tekauz (1985) was used. Numbers are mean of 3 replications. R, resistant; S, susceptible

Isolate numbers and the provinces where the isolates obtained

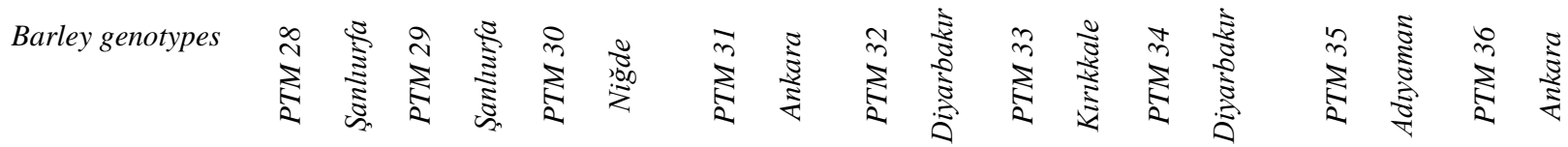

\begin{tabular}{|c|c|c|c|c|c|c|c|c|c|c|c|c|c|c|c|c|c|c|}
\hline 1 Arimont & 5 & $\mathrm{R}$ & 3 & $\mathrm{R}$ & 1 & $\mathrm{R}$ & 1 & $\mathrm{R}$ & 5 & $\mathrm{R}$ & 1 & $\mathrm{R}$ & 1 & $\mathrm{R}$ & 1 & $\mathrm{R}$ & 2 & $\mathrm{R}$ \\
\hline 2 Cape & 7 & $S$ & 5 & $\mathrm{R}$ & 1 & $\mathrm{R}$ & 1 & $\mathrm{R}$ & 5 & $\mathrm{R}$ & 1 & $\mathrm{R}$ & 1 & $\mathrm{R}$ & 2 & $\mathrm{R}$ & 1 & $\mathrm{R}$ \\
\hline 3 Chebec & 5 & $\mathrm{R}$ & 3 & $\mathrm{R}$ & 1 & $\mathrm{R}$ & 1 & $\mathrm{R}$ & 5 & $\mathrm{R}$ & 1 & $\mathrm{R}$ & 1 & $\mathrm{R}$ & 1 & $\mathrm{R}$ & 2 & $\mathrm{R}$ \\
\hline 4 CI3546 & 7 & $S$ & 3 & $\mathrm{R}$ & 1 & $\mathrm{R}$ & 2 & $\mathrm{R}$ & 3 & $\mathrm{R}$ & 1 & $\mathrm{R}$ & 2 & $\mathrm{R}$ & 3 & $\mathrm{R}$ & 1 & $\mathrm{R}$ \\
\hline 5 CI11458 & 7 & $S$ & 7 & $S$ & 2 & $\mathrm{R}$ & 1 & $\mathrm{R}$ & 5 & $\mathrm{R}$ & 2 & $\mathrm{R}$ & 1 & $\mathrm{R}$ & 1 & $\mathrm{R}$ & 2 & $\mathrm{R}$ \\
\hline 6 CI5286 & 5 & $\mathrm{R}$ & 3 & $\mathrm{R}$ & 2 & $\mathrm{R}$ & 1 & $\mathrm{R}$ & 3 & $\mathrm{R}$ & 1 & $\mathrm{R}$ & 1 & $\mathrm{R}$ & 2 & $\mathrm{R}$ & 1 & $\mathrm{R}$ \\
\hline 7 CI5791 & 7 & $\mathrm{~S}$ & 5 & $\mathrm{R}$ & 1 & $\mathrm{R}$ & 2 & $\mathrm{R}$ & 5 & $\mathrm{R}$ & 2 & $\mathrm{R}$ & 1 & $\mathrm{R}$ & 1 & $\mathrm{R}$ & 1 & $\mathrm{R}$ \\
\hline 8 CI7584 & 7 & $S$ & 5 & $\mathrm{R}$ & 1 & $\mathrm{R}$ & 1 & $\mathrm{R}$ & 5 & $\mathrm{R}$ & 2 & $\mathrm{R}$ & 2 & $\mathrm{R}$ & 2 & $\mathrm{R}$ & 1 & $\mathrm{R}$ \\
\hline 9 CI9214 & 7 & $\mathrm{~S}$ & 5 & $\mathrm{R}$ & 1 & $\mathrm{R}$ & 1 & $\mathrm{R}$ & 7 & $S$ & 2 & $\mathrm{R}$ & 1 & $\mathrm{R}$ & 1 & $\mathrm{R}$ & 1 & $\mathrm{R}$ \\
\hline 10 CI9776 & 5 & $\mathrm{R}$ & 5 & $\mathrm{R}$ & 1 & $\mathrm{R}$ & 1 & $\mathrm{R}$ & 5 & $\mathrm{R}$ & 2 & $\mathrm{R}$ & 1 & $\mathrm{R}$ & 1 & $\mathrm{R}$ & 2 & $\mathrm{R}$ \\
\hline 11 CI9819 & 5 & $\mathrm{R}$ & 5 & $\mathrm{R}$ & 1 & $\mathrm{R}$ & 2 & $\mathrm{R}$ & 5 & $\mathrm{R}$ & 2 & $\mathrm{R}$ & 1 & $\mathrm{R}$ & 2 & $\mathrm{R}$ & 1 & $\mathrm{R}$ \\
\hline 12 CI16150 & 5 & $\mathrm{R}$ & 5 & $\mathrm{R}$ & 1 & $\mathrm{R}$ & 1 & $\mathrm{R}$ & 3 & $\mathrm{R}$ & 1 & $\mathrm{R}$ & 1 & $\mathrm{R}$ & 2 & $\mathrm{R}$ & 2 & $\mathrm{R}$ \\
\hline 13 Galleon & 8 & $S$ & 5 & $\mathrm{R}$ & 2 & $\mathrm{R}$ & 1 & $\mathrm{R}$ & 5 & $\mathrm{R}$ & 2 & $\mathrm{R}$ & 2 & $\mathrm{R}$ & 2 & $\mathrm{R}$ & 2 & $\mathrm{R}$ \\
\hline 14 Haruna Nijo & 7 & $S$ & 5 & $\mathrm{R}$ & 1 & $\mathrm{R}$ & 1 & $\mathrm{R}$ & 5 & $\mathrm{R}$ & 2 & $\mathrm{R}$ & 2 & $\mathrm{R}$ & 2 & $\mathrm{R}$ & 2 & $\mathrm{R}$ \\
\hline 15 Keel & 7 & $S$ & 5 & $\mathrm{R}$ & 2 & $\mathrm{R}$ & 1 & $\mathrm{R}$ & 5 & $\mathrm{R}$ & 2 & $\mathrm{R}$ & 2 & $\mathrm{R}$ & 2 & $\mathrm{R}$ & 2 & $\mathrm{R}$ \\
\hline 16 Kombar & 7 & S & 5 & $\mathrm{R}$ & 1 & $\mathrm{R}$ & 1 & $\mathrm{R}$ & 5 & $\mathrm{R}$ & 2 & $\mathrm{R}$ & 1 & $\mathrm{R}$ & 1 & $\mathrm{R}$ & 2 & $\mathrm{R}$ \\
\hline 17 Skiff & 7 & $S$ & 3 & $\mathrm{R}$ & 1 & $\mathrm{R}$ & 1 & $\mathrm{R}$ & 5 & $\mathrm{R}$ & 2 & $\mathrm{R}$ & 2 & $\mathrm{R}$ & 2 & $\mathrm{R}$ & 2 & $\mathrm{R}$ \\
\hline 18 Steptoe & 7 & $S$ & 5 & $\mathrm{R}$ & 2 & $\mathrm{R}$ & 5 & $\mathrm{R}$ & 5 & $\mathrm{R}$ & 7 & $S$ & 5 & $\mathrm{R}$ & 2 & $\mathrm{R}$ & 3 & $\mathrm{R}$ \\
\hline 19 Stirling & 7 & $S$ & 5 & $\mathrm{R}$ & 1 & $\mathrm{R}$ & 2 & $\mathrm{R}$ & 5 & $\mathrm{R}$ & 2 & $\mathrm{R}$ & 2 & $\mathrm{R}$ & 2 & $\mathrm{R}$ & 1 & $\mathrm{R}$ \\
\hline 20 Summitt & 7 & S & 5 & $\mathrm{R}$ & 1 & $\mathrm{R}$ & 1 & $\mathrm{R}$ & 5 & $\mathrm{R}$ & 2 & $\mathrm{R}$ & 1 & $\mathrm{R}$ & 2 & $\mathrm{R}$ & 1 & $\mathrm{R}$ \\
\hline 21 Torrens & 7 & S & 5 & $\mathrm{R}$ & 1 & $\mathrm{R}$ & 1 & $\mathrm{R}$ & 5 & $\mathrm{R}$ & 2 & $\mathrm{R}$ & 1 & $\mathrm{R}$ & 2 & $\mathrm{R}$ & 1 & $\mathrm{R}$ \\
\hline 22 TR250 & 7 & $S$ & 3 & $\mathrm{R}$ & 1 & $\mathrm{R}$ & 1 & $\mathrm{R}$ & 5 & $\mathrm{R}$ & 1 & $\mathrm{R}$ & 1 & $\mathrm{R}$ & 1 & $\mathrm{R}$ & 1 & $\mathrm{R}$ \\
\hline 23 Bülbül 89 & 5 & $\mathrm{R}$ & 3 & $\mathrm{R}$ & 1 & $\mathrm{R}$ & 5 & $\mathrm{R}$ & 5 & $\mathrm{R}$ & 2 & $\mathrm{R}$ & 3 & $\mathrm{R}$ & 1 & $\mathrm{R}$ & 3 & $\mathrm{R}$ \\
\hline
\end{tabular}


Table 1 (Continued)- Response of barley differential genotypes to 45 Pyrenophora teres f. maculata isolates. For evaluation, a 1-9 scale developed by Tekauz (1985) was used. Numbers are mean of 3 replications. R, resistant; S, susceptible

Isolate numbers and the provinces where the isolates obtained

\begin{tabular}{|c|c|c|c|c|c|c|c|c|c|c|c|c|c|c|c|c|c|c|}
\hline $\begin{array}{l}\text { Barley } \\
\text { genotypes }\end{array}$ & $\underset{n}{\Sigma}$ & $\stackrel{\Xi}{\grave{\Xi}}$ & $\sum_{i}^{\infty}$ & 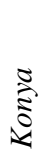 & $\underset{\Sigma}{\mathfrak{z}}$ & 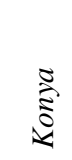 & 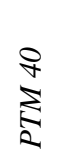 & 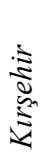 & 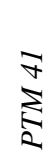 & 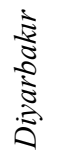 & 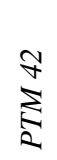 & $\begin{array}{l}\tilde{J} \\
\infty \\
\tilde{N} \\
\text { Dे }\end{array}$ & $\underset{\Sigma}{\gtrless}$ & 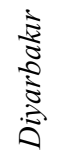 & $\underset{2}{j}$ & $\underset{\grave{\Xi}}{\grave{\Xi}}$ & $\underset{\mathbb{Z}}{\mathfrak{z}}$ & 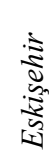 \\
\hline 1 Arimont & 3 & $\mathrm{R}$ & 1 & $\mathrm{R}$ & 1 & $\mathrm{R}$ & 1 & $\mathrm{R}$ & 7 & $\mathrm{~S}$ & 5 & $\mathrm{R}$ & 2 & $\mathrm{R}$ & 2 & $\mathrm{R}$ & 5 & $\mathrm{R}$ \\
\hline 2 Cape & 3 & $\mathrm{R}$ & 2 & $\mathrm{R}$ & 2 & $\mathrm{R}$ & 1 & $\mathrm{R}$ & 7 & $S$ & 5 & $\mathrm{R}$ & 1 & $\mathrm{R}$ & 3 & $\mathrm{R}$ & 3 & $\mathrm{R}$ \\
\hline 3 Chebec & 2 & $\mathrm{R}$ & 1 & $\mathrm{R}$ & 2 & $\mathrm{R}$ & 1 & $\mathrm{R}$ & 5 & $\mathrm{R}$ & 3 & $\mathrm{R}$ & 1 & $\mathrm{R}$ & 5 & $\mathrm{R}$ & 3 & $\mathrm{R}$ \\
\hline 4 CI3546 & 2 & $\mathrm{R}$ & 2 & $\mathrm{R}$ & 3 & $\mathrm{R}$ & 1 & $\mathrm{R}$ & 5 & $\mathrm{R}$ & 2 & $\mathrm{R}$ & 5 & $\mathrm{R}$ & 3 & $\mathrm{R}$ & 3 & $\mathrm{R}$ \\
\hline 5 CI11458 & 2 & $\mathrm{R}$ & 2 & $\mathrm{R}$ & 2 & $\mathrm{R}$ & 5 & $\mathrm{R}$ & 5 & $\mathrm{R}$ & 3 & $\mathrm{R}$ & 2 & $\mathrm{R}$ & 3 & $\mathrm{R}$ & 3 & $\mathrm{R}$ \\
\hline 6 CI5286 & 2 & $\mathrm{R}$ & 1 & $\mathrm{R}$ & 1 & $\mathrm{R}$ & 1 & $\mathrm{R}$ & 7 & S & 3 & $\mathrm{R}$ & 5 & $\mathrm{R}$ & 3 & $\mathrm{R}$ & 3 & $\mathrm{R}$ \\
\hline 7 CI5791 & 2 & $\mathrm{R}$ & 1 & $\mathrm{R}$ & 1 & $\mathrm{R}$ & 1 & $\mathrm{R}$ & 5 & $\mathrm{R}$ & 5 & $\mathrm{R}$ & 2 & $\mathrm{R}$ & 3 & $\mathrm{R}$ & 3 & $\mathrm{R}$ \\
\hline 8 CI7584 & 2 & $\mathrm{R}$ & 1 & $\mathrm{R}$ & 1 & $\mathrm{R}$ & 1 & $\mathrm{R}$ & 5 & $\mathrm{R}$ & 5 & $\mathrm{R}$ & 2 & $\mathrm{R}$ & 3 & $\mathrm{R}$ & 3 & $\mathrm{R}$ \\
\hline 9 CI9214 & 1 & $\mathrm{R}$ & 1 & $\mathrm{R}$ & 2 & $\mathrm{R}$ & 1 & $\mathrm{R}$ & 7 & $S$ & 2 & $\mathrm{R}$ & 2 & $\mathrm{R}$ & 3 & $\mathrm{R}$ & 5 & $\mathrm{R}$ \\
\hline $10 \mathrm{CI} 9776$ & 2 & $\mathrm{R}$ & 2 & $\mathrm{R}$ & 2 & $\mathrm{R}$ & 1 & $\mathrm{R}$ & 5 & $\mathrm{R}$ & 3 & $\mathrm{R}$ & 2 & $\mathrm{R}$ & 3 & $\mathrm{R}$ & 3 & $\mathrm{R}$ \\
\hline 11 CI9819 & 2 & $\mathrm{R}$ & 2 & $\mathrm{R}$ & 2 & $\mathrm{R}$ & 1 & $\mathrm{R}$ & 3 & $\mathrm{R}$ & 5 & $\mathrm{R}$ & 2 & $\mathrm{R}$ & 3 & $\mathrm{R}$ & 3 & $\mathrm{R}$ \\
\hline 12 CI16150 & 5 & $\mathrm{R}$ & 2 & $\mathrm{R}$ & 1 & $\mathrm{R}$ & 1 & $\mathrm{R}$ & 5 & $\mathrm{R}$ & 3 & $\mathrm{R}$ & 2 & $\mathrm{R}$ & 3 & $\mathrm{R}$ & 2 & $\mathrm{R}$ \\
\hline 13 Galleon & 2 & $\mathrm{R}$ & 2 & $\mathrm{R}$ & 2 & $\mathrm{R}$ & 1 & $\mathrm{R}$ & 7 & $\mathrm{~S}$ & 7 & S & 2 & $\mathrm{R}$ & 5 & $\mathrm{R}$ & 2 & $\mathrm{R}$ \\
\hline 14 Haruna Nijo & 2 & $\mathrm{R}$ & 1 & $\mathrm{R}$ & 2 & $\mathrm{R}$ & 2 & $\mathrm{R}$ & 7 & $\mathrm{~S}$ & 3 & $\mathrm{R}$ & 2 & $\mathrm{R}$ & 3 & $\mathrm{R}$ & 5 & $\mathrm{R}$ \\
\hline 15 Keel & 3 & $\mathrm{R}$ & 3 & $\mathrm{R}$ & 3 & $\mathrm{R}$ & 2 & $\mathrm{R}$ & 7 & $S$ & 5 & $\mathrm{R}$ & 3 & $\mathrm{R}$ & 7 & $\mathrm{~S}$ & 5 & $\mathrm{R}$ \\
\hline 16 Kombar & 2 & $\mathrm{R}$ & 1 & $\mathrm{R}$ & 3 & $\mathrm{R}$ & 1 & $\mathrm{R}$ & 7 & S & 5 & $\mathrm{R}$ & 1 & $\mathrm{R}$ & 5 & $\mathrm{R}$ & 3 & $\mathrm{R}$ \\
\hline 17 Skiff & 2 & $\mathrm{R}$ & 2 & $\mathrm{R}$ & 3 & $\mathrm{R}$ & 3 & $\mathrm{R}$ & 5 & $\mathrm{R}$ & 5 & $\mathrm{R}$ & 2 & $\mathrm{R}$ & 3 & $\mathrm{R}$ & 5 & $\mathrm{R}$ \\
\hline 18 Steptoe & 7 & $S$ & 7 & $S$ & 7 & S & 5 & $\mathrm{R}$ & 7 & S & 7 & $S$ & 2 & $\mathrm{R}$ & 7 & $\mathrm{~S}$ & 7 & $\mathrm{~S}$ \\
\hline 19 Stirling & 2 & $\mathrm{R}$ & 2 & $\mathrm{R}$ & 1 & $\mathrm{R}$ & 1 & $\mathrm{R}$ & 7 & S & 7 & S & 3 & $\mathrm{R}$ & 5 & $\mathrm{R}$ & 3 & $\mathrm{R}$ \\
\hline 20 Summitt & 2 & $\mathrm{R}$ & 2 & $\mathrm{R}$ & 2 & $\mathrm{R}$ & 1 & $\mathrm{R}$ & 7 & $\mathrm{~S}$ & 3 & $\mathrm{R}$ & 3 & $\mathrm{R}$ & 5 & $\mathrm{R}$ & 3 & $\mathrm{R}$ \\
\hline 21 Torrens & 1 & $\mathrm{R}$ & 2 & $\mathrm{R}$ & 1 & $\mathrm{R}$ & 1 & $\mathrm{R}$ & 8 & S & 5 & $\mathrm{R}$ & 2 & $\mathrm{R}$ & 3 & $\mathrm{R}$ & 3 & $\mathrm{R}$ \\
\hline 22 TR250 & 2 & $\mathrm{R}$ & 1 & $\mathrm{R}$ & 1 & $\mathrm{R}$ & 1 & $\mathrm{R}$ & 7 & $\mathrm{~S}$ & 3 & $\mathrm{R}$ & 1 & $\mathrm{R}$ & 3 & $\mathrm{R}$ & 3 & $\mathrm{R}$ \\
\hline 23 Bülbül 89 & 7 & $S$ & 7 & $S$ & 7 & S & 5 & $\mathrm{R}$ & 8 & S & 5 & $\mathrm{R}$ & 3 & $\mathrm{R}$ & 5 & $\mathrm{R}$ & 7 & $S$ \\
\hline
\end{tabular}


Table 2- Nineteen pathotypes of Pyrenophora teres $\mathrm{f}$. maculata using a differential set containing 23 barley genotypes and isolate locations

\begin{tabular}{|c|c|c|c|c|}
\hline Isolates & Locations & $\begin{array}{l}\text { Genotype numbers } \\
\text { showing susceptible } \\
\text { reactions/ Pathotype numbers }\end{array}$ & genotypes & $\begin{array}{l}\text { Virulence } \\
\text { value }\end{array}$ \\
\hline PTM 30 & Niğde & Pathotype 0 & 0 & 1.17 \\
\hline PTM 34 & Diyarbakır & & & 1.47 \\
\hline PTM 31 & Ankara & & & 1.52 \\
\hline PTM 9 & Eskişehir & & & 1.56 \\
\hline PTM 36 & Ankara & & & 1.60 \\
\hline PTM 35 & Adiyaman & & & 1.65 \\
\hline PTM 14 & Konya & & & 1.69 \\
\hline PTM 40 & Kirşehir & & & 1.69 \\
\hline PTM 7 & Diyarbakır & & & 1.73 \\
\hline PTM 8 & Şanlıurfa & & & 1.73 \\
\hline PTM 22 & Kayseri & & & 1.78 \\
\hline PTM 25 & Afyonkarahisar & & & 1.86 \\
\hline PTM 2 & Şanlıurfa & & & 1.95 \\
\hline PTM 19 & Eskişehir & & & 2.17 \\
\hline PTM 43 & Diyarbakır & & & 2.26 \\
\hline PTM 21 & Kahramanmaraş & & & 3.60 \\
\hline TM 20 & Diyarbakır & & & 4.04 \\
\hline PTM 33 & Kirıkkale & Pathotype 18 & 1 & 1.91 \\
\hline PTM 10 & Kayseri & Pathotype 23 & 1 & 1.82 \\
\hline PTM 24 & Kirşehir & & & 2.13 \\
\hline PTM 1 & Konya & & & 3.04 \\
\hline PTM 3 & Ankara & Pathotype 23 & 1 & 3.17 \\
\hline PTM 5 & Aksaray & & & 3.34 \\
\hline PTM 29 & Şanlıurfa & Pathotype 5 & 1 & 4.47 \\
\hline PTM 32 & Diyarbakır & Pathotype 9 & 1 & 4.82 \\
\hline PTM 38 & Konya & Pathotype 18-23 & 2 & 2.08 \\
\hline PTM 39 & Konya & & & 2.26 \\
\hline PTM 37 & Konya & & & 2.60 \\
\hline PTM 15 & Ankara & & & 3.17 \\
\hline PTM 45 & Eskișehir & & & 3.69 \\
\hline PTM 23 & Çankırı & & & 3.78 \\
\hline PTM 16 & Sivas & & & 3.91 \\
\hline PTM 44 & Konya & Pathotype 15-18 & 2 & 3.82 \\
\hline PTM 42 & Yozgat & Pathotype 13-18-19 & 3 & 4.30 \\
\hline PTM 4 & Kahramanmaraş & Pathotype 5-20-23 & 3 & 5 \\
\hline PTM 27 & Çankırı & Pathotype 15-16-18-23 & 4 & 4.39 \\
\hline PTM 6 & Mardin & Pathotype 5-14-18-19 & 4 & 4.47 \\
\hline PTM 18 & Ankara & Pathotype 10-13-18-23 & 4 & 4.47 \\
\hline PTM 12 & Kilis & Pathotype 5-14-16-19-20 & 5 & 5.08 \\
\hline \multicolumn{5}{|l|}{ Hordeum } \\
\hline \multicolumn{5}{|l|}{ spontaneum } \\
\hline PTM 11 & Mardin & Pathotype 2-4-5-16-19-20-23 & 7 & 5.13 \\
\hline PTM 26 & Edirne & $\begin{array}{l}\text { Pathotype 2-4-5-10-13-14-16- } \\
18-19-20-21\end{array}$ & 11 & 6 \\
\hline PTM 41 & Diyarbakır & $\begin{array}{l}\text { Pathotype 1-2-6-9-13-14-15-16-18- } \\
19-20-21-22-23\end{array}$ & 14 & 6.21 \\
\hline PTM 28 & Şanlıurfa & $\begin{array}{l}\text { Pathotype } 2-4-5-7-8-9-13-14-15-16- \\
17-18-19-20-21-22\end{array}$ & 16 & 6.43 \\
\hline PTM 13 & Gaziantep & $\begin{array}{l}\text { Pathotype 1-3-5-7-10-11-12-13-14- } \\
15-16-17-18-19-20-23\end{array}$ & 16 & 6.65 \\
\hline PTM 17 & Şanlıurfa & Pathotype 1-2-3-4-5-6-7-8-9-10-11-12-13-14-15- & 23 & 7.47 \\
\hline Hordeum & & $16-17-18-19-20-21-22-23$ & & \\
\hline spontaneum & & & & \\
\hline
\end{tabular}


Genotypes CI3576, CI9214, CI9776 and Torrens exhibited resistant reactions to $91 \%$ of the isolates in our current study. These genotypes showed susceptible reactions to 4 isolates. Akhavan et al (2016) reported that CI9214 genotype was resistant to all Ptm isolates except two. In another study, genotypes CI9214 and CI9776 showed a resistant reaction to all isolates used (Karki \& Sharp 1986). Differential cultivar Torrens exhibited moderately resistant-moderately susceptible reactions (McLean et al 2012) and different infection responses among the isolates were observed (McLean et al 2014a).

In our current study, Arimont, CI5791, Skiff and TR250 genotypes exhibited resistant reactions to 42 isolates (93\%). These genotypes showed susceptible reactions to 3 isolates. Akhavan et al (2016) reported the virulence of 19 (70.4\%) Ptm isolates on genotype CI5791. Cultivar Arimont was reported as susceptible in a previous study (Karki \& Sharp 1986). Cultivar Skiff was reported as generally moderately resistant and genotype TR250 was reported as moderately susceptible (McLean et al 2012).

Cape, Keel, Galleon, Haruna Nijo, Kombar, Summitt, CI11458 and Stirling genotypes exhibited low infection responses to $88 \%, 86 \%, 84 \%, 84 \%, 82 \%, 82 \%, 80 \%$ and $80 \%$ of the isolates, respectively, in our current study. In other studies, cultivar Keel was found resistant to all isolates, however, genotypes Cape, CI11458 and Summitt were moderately susceptible and cultivar Galleon was moderately resistant. Cultivar Kombar exhibited a susceptible reaction to more than half of the isolates (McLean et al 2012, 2014a). Cultivar Stirling showed different reactions to different isolates (Gupta et al 2012).

In our current study, cultivar Steptoe was susceptible to $40 \%$ of the isolates. In Akhavan et al (2016) study, this cultivar was susceptible to $81.5 \%$ of the isolates.

Barley cultivars and genotypes Cape, CI11458, CI5791, CI7584, CI9819, Kombar and Bülbül 89 were also used in Çelik Oğuz \& Karakaya (2017) study. In their study, genotypes Cape, CI11458, CI5791, CI7584, CI9819, Kombar and Bülbül 89 showed susceptible reactions to 10,16, 9, 13, 10, 20 and 44 out of 50 isolates, respectively. In our current study, genotypes Cape, CI11458, CI5791, CI7584, CI9819, Kombar and Bülbül 89 exhibited susceptible reactions to 5, $9,3,2,2,8$ and 19 out of 45 isolates, respectively.

In the current study, cultivars Steptoe and Bülbül 89 exhibited susceptible reactions to 18 and 19 isolates, respectively. These cultivars were the most susceptible cultivars. Cultivar Bülbül 89 could be used as universal susceptible genotype in an international Ptm differential set. The genotypes used in this study were useful in differentiating Ptm pathotypes.

\section{Conclusions}

For determination of Pyrenophora teres f. maculata pathotypes, a differential set consisted of 22 international cultivars and genotypes and a susceptible Turkish barley variety Bülbül 89 were tested using 45 isolates obtained from different regions of Turkey. Nineteen pathotypes were determined out of 45 isolates used. Cultivar Bülbül 89 could be used as universal susceptible genotype in an international Ptm differential set. The genotypes used in this study were useful in differentiating Ptm pathotypes.

\section{Acknowledgement}

We thank Mark S. McLean (Agriculture Victoria, Horsham Vic 3401, Australia) for providing the barley differential seeds used in this study.

\section{References}

Akhavan A, Turkington T K, Askarian H, Tekauz A, Xi K, Tucker J R, Kutcher H R \& Strelkov S E (2016). Virulence of Pyrenophora teres populations in western Canada. Canadian Journal of Plant Pathology 38 (2): 183-196

Aktaş H (1995). Reaction of Turkish and German barley varieties and lines to the virulent strain T4 of Pyrenophora teres. Rachis 14: $9-13$ 
Aktaş H (2001). Önemli hububat hastalıkları ve sürvey yöntemleri. T.C. Tarım ve Köyişleri Bakanlığı. Tarımsal Araştırmalar Genel Müdürlügü. Bitki Sağlığı Araştırmaları Daire Başkanlığı, Ankara

Boungab K, Belabid L, Fortas Z \& Bayaa B (2012). Pathotype diversity among Algerian isolates of Pyrenophora teres f. teres. Phytopathologia Mediterranea 51(3): 577-586

Çelik Oğuz A \& Karakaya A (2017). Pathotypes of Pyrenophora teres on barley in Turkey. Phytopathologia Mediterranea 56(2): 224-234

Grewal T S, Rossnagel B G \& Scoles G J (2008). The utility of molecular markers for barley net blotch resistance across geographic regions. Crop Science 48: 2321-2333

Gupta S, Loughman R, D’Antuono M \& Bradley J (2012). Characterisation and diversity of Pyrenophora teres f. maculata isolates in Western Australia. Australasian Plant Pathology 41(1): 31-40

Karakaya A, Mert Z, Çelik Oğuz A, Azamparsa M R, Çelik E, Akan K \& Çetin L (2014). Current status of scald and net blotch diseases of barley in Turkey. In: IWBLD-1 ${ }^{\text {st }}$ International Workshop on Barley Leaf Diseases, 3-6 June, Salsomaggiore Terme, Italy

Karki C B \& Sharp E L (1986). Pathogenic variation in some isolates of Pyrenophora teres f. maculata on barley. Plant Disease 70 : 684-687

Liu Z, Ellwood S R, Oliver R P \& Friesen T L (2011). Pyrenophora teres: profile of an increasingly damaging barley pathogen. Molecular Plant Pathology 12(1): 1-19

Mathre D E (Ed.) (1982). Compendium of Barley Diseases. American Phytopathological Society, Minnesota pp. 78

McLean M S, Howlett B J \& Hollaway G J (2009). Epidemiology and control of spot form of net blotch (Pyrenophora teres f. maculata) of barley: a review. Crop and Pasture Science 60(4): 303-315

McLean M S, Howlett B J, Turkington T K, Platz G J \& Hollaway G J (2012). Spot form of net blotch resistance in a diverse set of barley lines in Australia and Canada. Plant Disease 96(4): 569-576

McLean M S, Martin A, Gupta S, Sutherland M W, Hollaway G J \& Platz, G J (2014a). Validation of a new spot form of net blotch differential set and evidence for hybridisation between the spot and net forms of net blotch in Australia. Australasian Plant Pathology 43(3): 223-233

McLean M S, Turkington T K, Jalli M, Smit F \& Platz G J (2014b). A new international differential set for testing Pyrenophora teres f. maculata. In: IWBLD-1 st International Workshop on Barley Leaf Diseases, 3-6 June, Salsomaggiore Terme, Italy

Tekauz A (1985). A numerical scale to classify reactions of barley to Pyrenophora teres. Canadian Journal of Plant Pathology 7(2): $181-183$

Tekauz A (1990). Characterization and distribution of pathogenic variation in Pyrenophora teres f. teres and P. teres f. maculata from western Canada. Canadian Journal of Plant Pathology 12(2): 141-148

Wu H L, Steffenson B J, Li Y, Oleson A E \& Zhong S (2003). Genetic variation for virulence and RFLP markers in Pyrenophora teres. Canadian Journal of Plant Pathology 25(1): 82-90

Zadoks J C, Chang T T \& Konzak C F (1974). A decimal code for the growth stages of cereals. Weed Research 14: 415- 421 